\title{
Desirable Characteristics of Hepatitis C Treatment Regimens: A Review of What We Have and What We Need
}

\author{
Monique R. Bidell • Milena McLaughlin · John Faragon • \\ Caroline Morse $\cdot$ Nimish Patel (D)
}

Received: March 17, 2016/Published online: July 6, 2016

(C) The Author(s) 2016. This article is published with open access at Springerlink.com

\section{ABSTRACT}

There have been dramatic advancements in the treatment of chronic hepatitis C (HCV) infection. This is largely due to the approval of several direct-acting antiviral agents (DAAs) from a variety of medication classes with novel mechanisms of action. These therapies are a welcomed advancement given their improved efficacy and tolerability compared to pegylated interferon and ribavirin (RBV)-based regimens. These convenient, all-oral regimens

Enhanced content To view enhanced content for this article go to http://www.medengine.com/Redeem/ EED4F06065AA9F60.

M. R. Bidell · C. Morse · N. Patel $(\square)$

Department of Pharmacy Practice, Albany College of Pharmacy and Health Sciences, 106 New Scotland Avenue, Albany, NY 12208, USA

e-mail: Nimish.patel@acphs.edu

\section{McLaughlin}

Department of Pharmacy Practice, Midwestern University Chicago College of Pharmacy, 555 31st St, Downers Grove, Downers Grove, IL 60515, USA

M. McLaughlin

Department of Pharmacy, Northwestern Memorial

Hospital, 251 E Huron St, Chicago, IL 60611, USA

J. Faragon

Division of HIV Medicine, Albany Medical College,

43 New Scotland Avenue, Albany, NY 12208, USA treat a variety of genotypes and often offer high cure rates in a variety of $\mathrm{HCV}$-infected populations. While there are several benefits associated with these therapies, there are also notable shortcomings. Shortcomings include diminished response or need for adjunctive RBV in difficult-to-treat populations (decompensated cirrhosis, active substance abuse patients, advanced kidney disease, etc.), activity against select genotypes, substantial drug-drug interaction potential, and high cost. Therefore, while current DAA-based therapies have several favorable attributes, each also has its limitations. The purpose of this review is to (1) identify the characteristics of an ideal HCV treatment regimen, (2) describe desirable features of existing regimens, (3) summarize limitations of existing regimens, and (4) introduce promising emerging therapies. This manuscript will serve as a guide for evaluating the caliber of future HCV treatment regimens.

Keywords: Effectiveness; Genotype; Hepatitis C; Pharmacotherapy; Response; Safety; Treatment 


\section{INTRODUCTION}

The emergence of direct-acting antiviral agents (DAAs) has dramatically transformed the chronic hepatitis C (HCV) treatment landscape. Compared to the historic regimen of pegylated interferon (PEG-IFN) and ribavirin (RBV), DAAs exhibit both increased tolerability and efficacy. Anticipated frequencies of sustained virologic response (SVR12), defined as an undetectable HCV RNA viral load at 12 weeks after completion of therapy, are now $>90 \%$ for many DAA-containing therapies [1]. Achievement of SVR is associated with numerous health benefits including regression of fibrosis, a substantial reduction in the risk of hepatocellular carcinoma, and a 90\% reduction in liver-related mortality [1]. Despite these benefits, only about $5 \%$ of the estimated 2.2-3.2 million Americans infected with chronic HCV (though nearly half are unaware of their diagnosis) receive treatment $[1,2]$. While current therapies are highly efficacious and effective, many are extremely patient-specific and treatment selection is driven by viral genotype, presence of cirrhosis, use of concomitant medications, and many other considerations. They are also costly and may not be accessible to all patients. Therefore, while the approval of the DAAs is a welcomed advancement compared to therapies containing PEG-IFN and RBV, there are severable desirable traits of an "ideal" HCV therapy that have yet to be possessed by a single regimen. Emergence of this highly-desirable therapy would mean a step closer to HCV control and elimination in the United States. The purpose of this review is to (1) identify the characteristics of an ideal HCV treatment regimen, (2) describe desirable features of existing regimens, (3) summarize limitations of existing regimens, and (4) present promising emerging therapies. This review will discuss ledipasvir/sofosbuvir (LDV/ SOF), paritaprevir/ritonavir/ombitasvir/dasabuvir (PrOD), simeprevir/sofosbuvir (SIM/SOF), daclatasvir/sofosbuvir (DAC/SOF), and grazoprevir/elbasvir (GZR/EBR). Given its similarity to PrOD, PrO will not be discussed [3]. This article is based on previously conducted studies and does not involve any new studies of human or animal subjects performed by any of the authors.

\section{EFFICACIOUS AND EFFECTIVE}

While intuitive, an ideal HCV regimen should be one that demonstrates high efficacy and effectiveness. Cure of infection is defined as achievement of sustained virologic response (SVR), or undetectable HCV RNA viral load, several weeks post-therapy completion. Historically, cure was assessed at 6 months (SVR24) after completion of up to 48 weeks of therapy. Considering that assessment at 12 weeks post-therapy completion has shown to be equally relevant [4], and that many contemporary treatment regimens are only $8-12$ weeks in duration, SVR12 is the current standard [5].

Several available DAA-containing therapies have demonstrated impressive frequencies of SVR12, often greater than $90 \%$ and approaching $100 \%$, in clinical trials [1]. SVR12 rates of currently available regimens in clinical trials are provided in Table 1. Though these findings are important for market approval, trial populations may not be fully representative of patients who will receive the treatment in practice. Therefore, an ideal treatment regimen should demonstrate not only high efficacy but also strong potential for real-world effectiveness. Favorable outcomes should be demonstrated across a spectrum of HCV-infected patients, including those who 


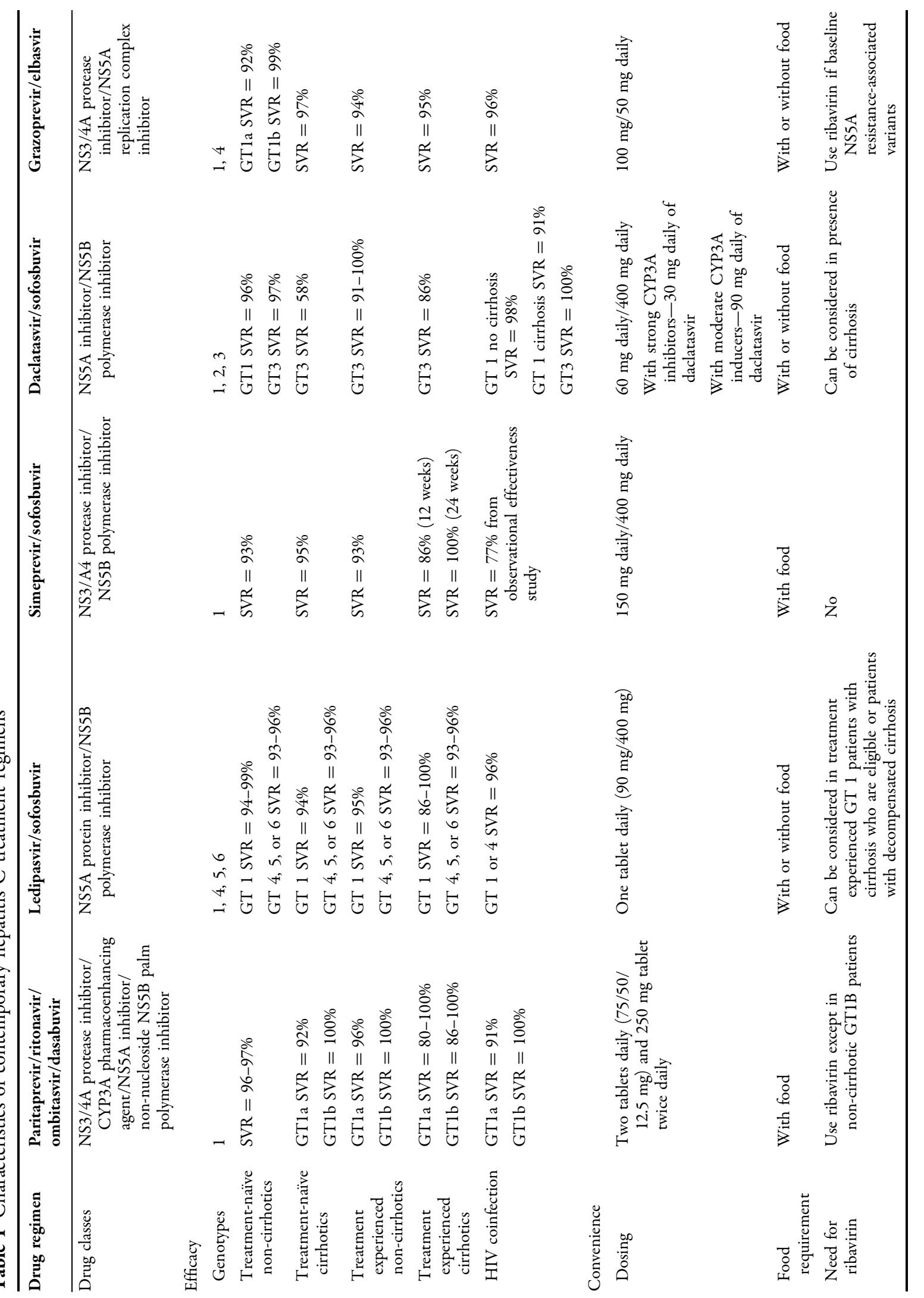




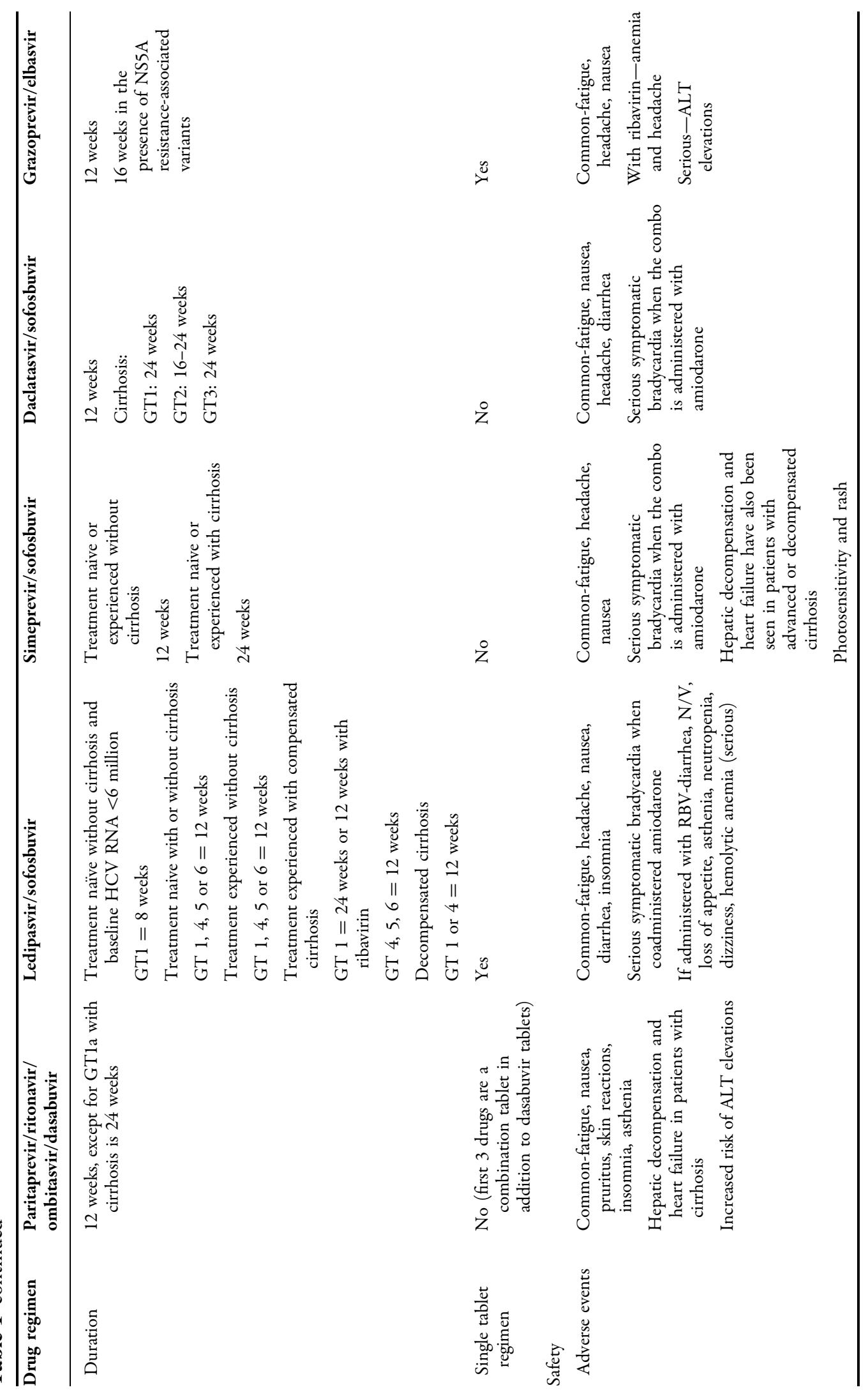




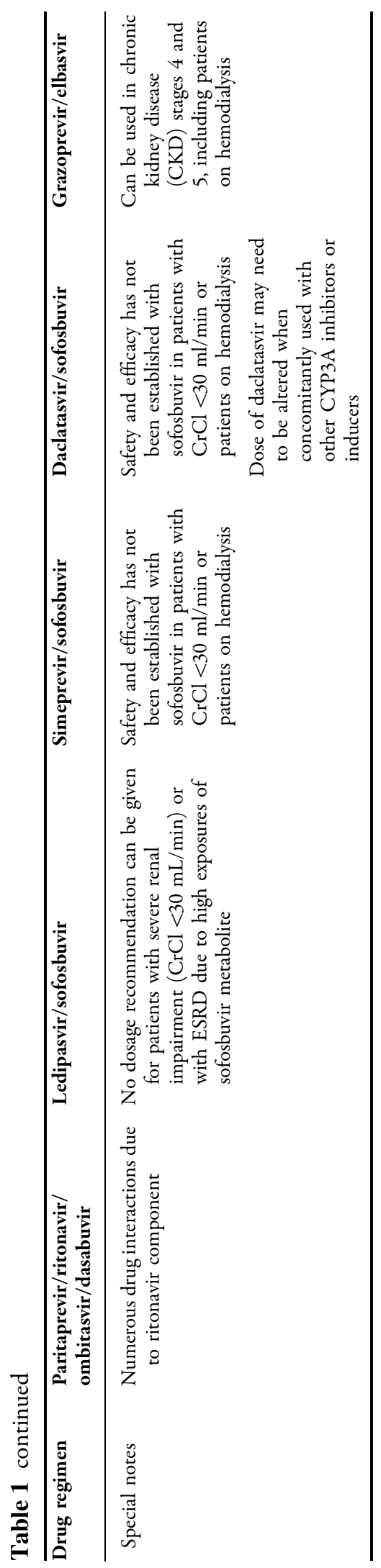

are relatively healthy and treatment-naïve ("uncomplicated") as well as those considered more difficult-to-treat or "complicated" based on individual history and comorbidities. These patients may be treatment-experienced, with high baseline viral loads and genetic variants (e.g., Q80K in the context of simeprevir-containing regimens), have various coinfections such as human immunodeficiency virus (HIV) or hepatitis B, and/or have advanced liver disease (e.g., decompensated cirrhosis). Historically, these patients have diminished treatment responses and higher risks of $\mathrm{HCV}$-associated complications relative to treatment-naïve, HCV mono-infected patients with no evidence of liver damage or cirrhosis [1].

Consistent with expected outcomes from the ideal treatment regimen, several available therapies have demonstrated substantial efficacy in difficult-to-treat patients, though adjunctive RBV is often required. Cure rates with DAA-based therapies, which often exceed $90 \%$, are staggering compared to those associated with PEG-IFN and RBV treatment, which were approximately $17 \%$ for cirrhotic patients, for example [6].

However, probability of cure remains highly patient- and regimen-specific (e.g., presence of the Q80K mutation in GT1a-infected, treatment-experienced, cirrhotic patients is associated with failure to SIM/SOF) [7, 8]. Several populations face limited treatment options, including those with less common GTs, renal disease, pregnant women, post-transplant recipients, and previous DAA-based therapy failures [1]. Additionally, treatment would still prove successful in populations unlikely to be included in trials or large studies, including those with a recent history of substance use, advanced age, and psychiatric illness. Despite numerous 
patient-specific challenges that complicate effectiveness of available HCV therapies, the ideal treatment regimen would result in high probability of SVR12 consistently across all HCV-infected populations without need for adjunctive medications such as RBV.

\section{SAFE}

\section{Non-Toxic}

HCV treatment-associated toxicity was a considerable patient care hurdle prior to 2013 when PEG-IFN and RBV continued to be included in the mainstay of treatment. Given its association with several adverse effects and laboratory abnormalities, PEG-IFN plus RBV required close safety monitoring. In some cases, supplementary medications were required to treat or manage $\mathrm{HCV}$ treatment-associated adverse effects (e.g., epoetin alfa to treat drug-induced anemia). In a contemporary setting, an ideal HCV treatment should be tolerable and unlikely to cause laboratory abnormalities. The latter is important as frequent laboratory testing during and potentially post-treatment are inconvenient and costly. Lack of both tolerability and overall treatment safety may lead to possible patient harm, premature discontinuation of therapy, and/or poor adherence leading to unsuccessful cure.

Most contemporary $\mathrm{HCV}$ regimens demonstrated high tolerability in clinical trials, with infrequent therapy discontinuations from serious adverse events. For DAC/SOF and LDV/SOF, the most commonly reported adverse events were minor, including fatigue and headache $[9,10]$. For SIM/SOF, a unique adverse effect is a variety of dermatologic manifestations including rash and pruritus. This typically occurs within
4 weeks of therapy initiation and may be due to certain drug chemical properties (e.g., SIM has a sulfa-like moiety) and/or photosensitivity potential [7]. PrOD, though generally well-tolerated in clinical studies, may cause serious hepatic injury. Patients with advanced liver disease appear to be particularly susceptible, as described in a recently issued FDA warning $[3,11]$. Additionally, PrOD is often co-administered with RBV, which can cause hemolytic anemia. Considering the toxicity potential of many currently available $\mathrm{HCV}$ therapies, an ideal treatment regimen would have a favorable toxicity profile with minimal risk of serious adverse events.

\section{Devoid of Drug-Drug Interactions}

While adverse effects of each regimen should be considered, it is also important to assess the safety of anti-HCV agents when given concomitantly with other medications. Drug-drug interactions (DDIs) are of substantial concern in the HCV-infected population, given that treatment regimens consist of multiple treatment medications for patients that frequently have medically managed comorbidities [12]. Many interactions involve the cytochrome (CYP) P450 isoenzyme system, including CYP3A4, which metabolizes DAAs and several other classes of medications [7]. Among the HCV regimens, SIM is an inhibitor of intestinal CYP3A4 and the ritonavir component of PrOD is involved in the hepatic inhibition of CYP3A4 as well as several other CYP isoenzymes [7, 11]. GZR is a weak inhibitor of CYP3A4 and may be implicated in fewer interactions [13]. LDV and EBR are inhibitors of p-glycoprotein (PGP) and breast cancer receptor protein (BCRP) [9, 13]. DAC is an inhibitor of PGP and organic anion transporter protein (OATP) 1B1 [10]. 
DDIs involving each of the HCV regimens and common classes of medications are displayed in Table 2. HMG-CoA reductase inhibitors (statins) are a popular medication class subject to numerous DDIs with HCV therapies, and management is agent-specific. One of the most serious DDIs identified with DAAs is coadministration of SOF and amiodarone, which can result in severe bradyarrhythmias. Coadministration is contra-indicated and patients using amiodarone should avoid SOF-containing therapy $[9,14-16]$.

Within the HIV/HCV co-infected population, DDIs with antiretrovirals are of particular concern. Most protease inhibitors (PIs) and non-nucleoside reverse transcriptase inhibitors (NNRTIs) are problematic for GZR/ EBR, SIM/SOF and PrOD [7, 11, 13]. For LDV/ SOF, increased exposure (AUC) to tenofovir disoproxil fumarate (TDF) is observed in HIV/ HCV co-infected patients using efavirenz or protease-inhibitor containing regimens [9]. However, the degree of enhanced exposure observed with efavirenz and TDF coadministration with $\mathrm{LDV} / \mathrm{SOF}$ is still within the range of tenofovir AUC values in which safety data exist. For regimens containing PIs and TDF that are coadministered with LDV/ SOF, the upper bound of the confidence interval slightly exceeds the range of tenofovir AUC values in which safety data exist. The future impact of the TDF interaction is unknown as use of tenofovir alafenamide (TAF) becomes more widespread. Dolutegravir and raltegravir appear to be the safest options for coadministration with HCV therapy. When considering DDI management, a key limitation of many available HCV therapies is the coformulation of multiple antivirals in a single product. Currently, dose personalization/ adjustment of individual agents associated with an interaction or toxicity within a co-formulated product is unfeasible. As currently available HCV therapies pose notable risks for potentially serious DDIs, elimination of this potential would be an important attribute of an ideal treatment regimen.

\section{CONVENIENT}

An ideal HCV treatment regimen would have convenient all oral administration. Reducing pill burden and decreasing regimen complexity are associated with improved clinical outcomes in other therapeutic domains and may extend to HCV [17-19]. In the HIV-infected population, use of single tablet regimens (STRs) is associated with improved medication adherence to antiretroviral therapy and decreased hospitalizations [20-22]. Similar conclusions cannot be made in the context of $\mathrm{HCV}$ infection, as studies have not yet been performed comparing medication adherence to single- versus multiple-tablet regimens and the effect of number of tablets per day on achieving SVR. In HCV, a single oral tablet formulation dosed infrequently (e.g., daily) for a short treatment duration would appear most desirable, largely due to convenience. However, the convenience of a single-tablet regimen needs to be tempered with a discussion of the relationship between non-adherence and resistance. It is unclear if non-adherence to a single-tablet regimen results in a higher potential for development of drug resistance than multiple-tablet regimens dosed multiple times per day. A thorough understanding of the pharmacokinetic/pharmacodynamics indices associated with the development of resistance will be imperative and predominantly applicable to missing doses of medications with short half-lives. This is an important area 


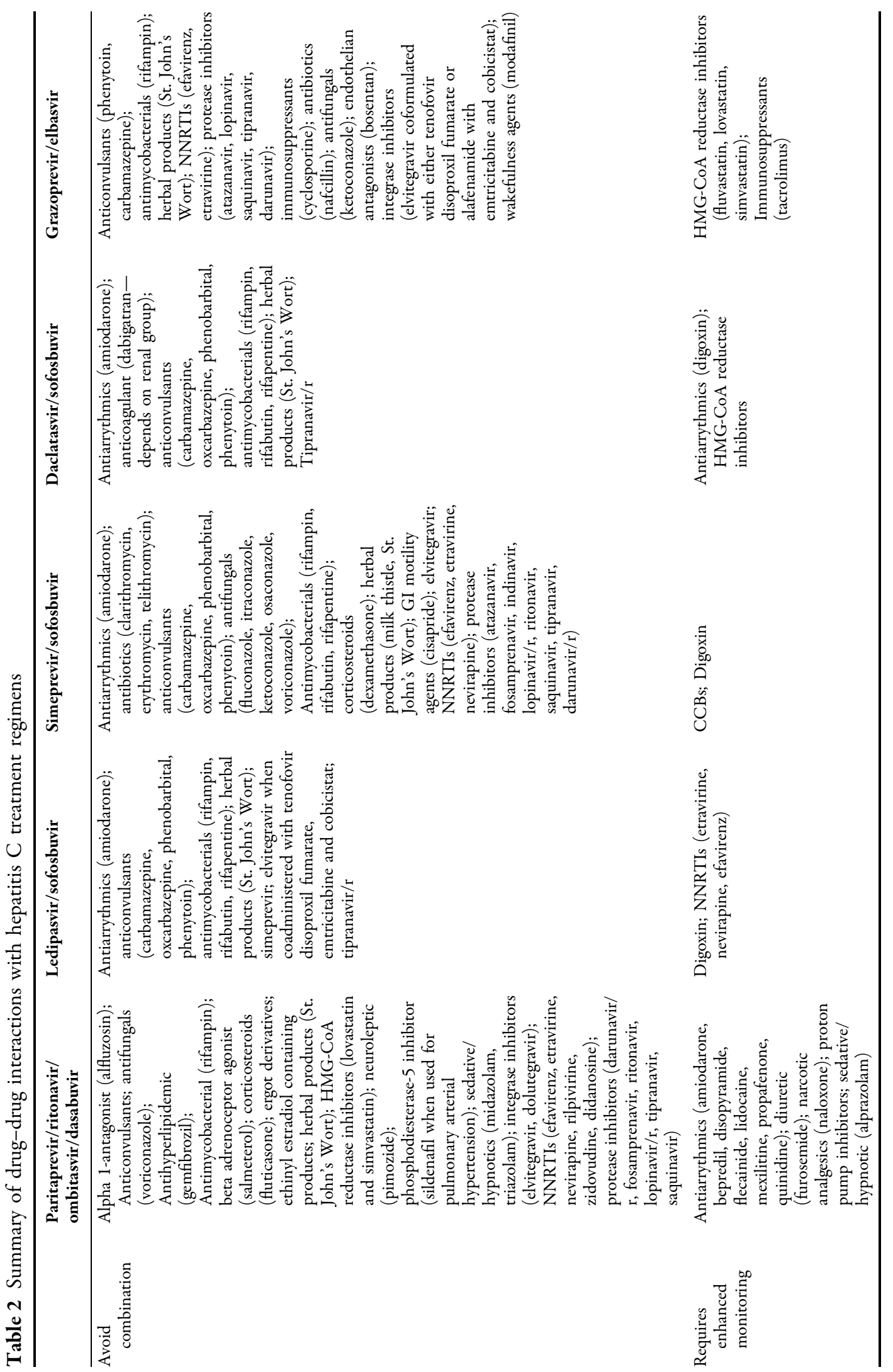




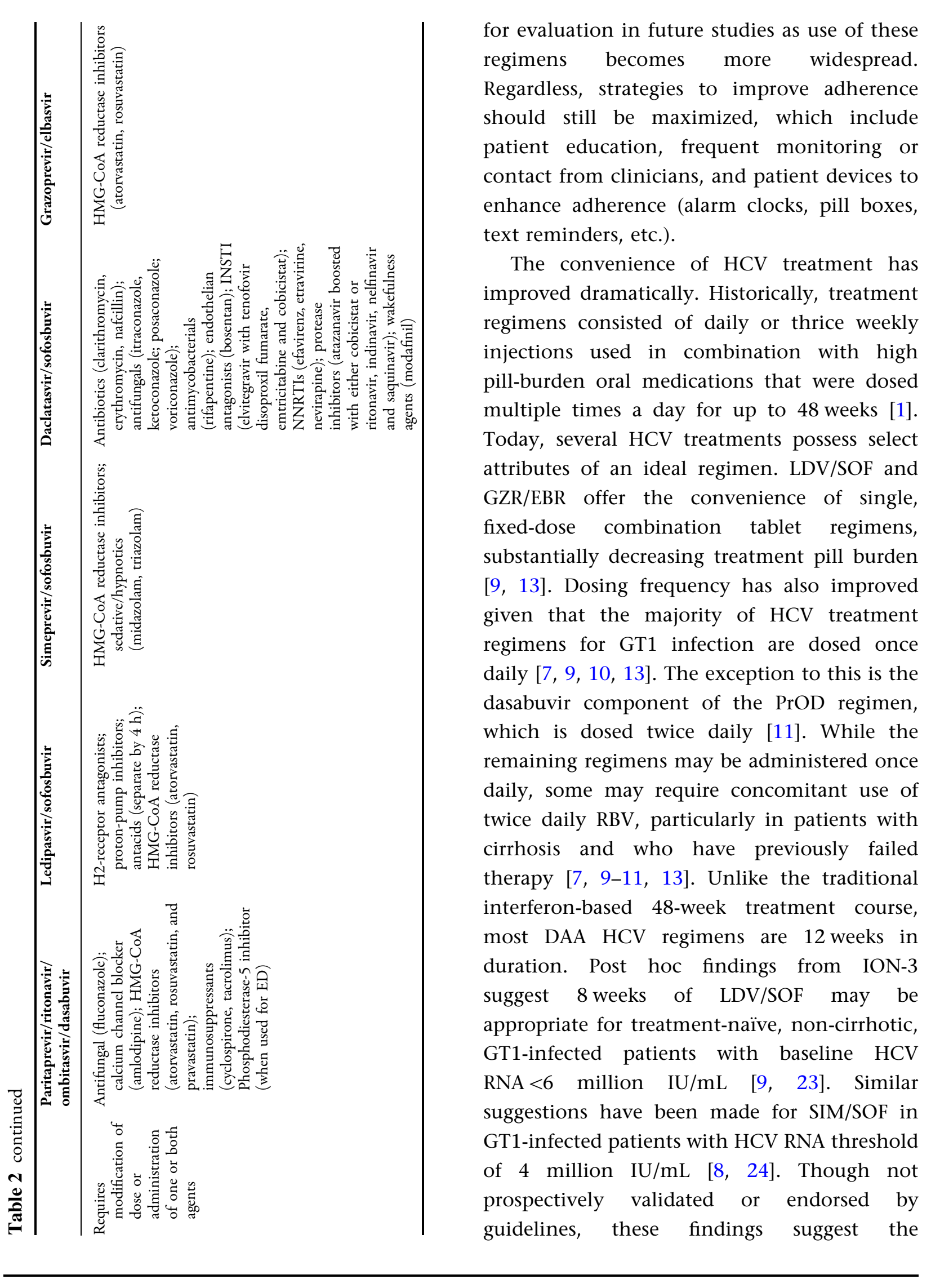


possibility of shorter treatment courses without compromised efficacy for select populations. GZR/EBR plus RBV for 8 weeks may offer another abbreviated treatment option, though SVR rates were $<90 \%$ [25]. Ideally, future regimens will offer a short course of conveniently administered therapy for all $\mathrm{HCV}$ patient populations.

\section{ACCESSIBLE AND AFFORDABLE}

An ideal $\mathrm{HCV}$ regimen will be one that is affordable and relatively easy to obtain for patients from all socioeconomic backgrounds. Many of these therapies are offered through patient assistance programs to increase accessibility and affordability for qualifying individuals. However, many patients with $\mathrm{HCV}$ infection face numerous barriers hampering access to optimal therapy [26]. In the US, barriers include high treatment costs, lack of third party payer coverage or coverage contingencies, requirement for prior authorization approval, and therapy restriction to only patients with severe infection. These limitations and restrictions greatly complicate patient access to appropriate $\mathrm{HCV}$ treatment. This is particularly concerning given the evidence that delays in therapy are associated with an increased risk of adverse HCV-associated outcomes [1].

The most widely discussed barrier is the high treatment cost, which may not be affordable out-of-pocket for the vast number of $\mathrm{HCV}$-infected patients that are uninsured or underinsured [13]. While the true cost of these medications to third party payers is largely unknown due to proprietary contract pricing, average wholesale pricing of many 12-week courses of DAA treatments are in excess of US\$90,000 for the medication alone (i.e. monitoring and clinic visit costs are not included in this price) [10]. Currently, the least expensive regimen is the newly-approved GZR/EBR, costing approximately US\$55,000 [11]. For those with prescription insurance, high copayments or deductibles may still exist. Additionally, while manufacturer-based patient assistance programs exist for the DAAs, some are associated with income restrictions or manufacturer specific guidelines for treatment [27].

If a patient does have prescription insurance, various restrictions to DAA coverage may apply. One restriction is prior authorization (PA), whereby clinicians must provide written justification to a third party payer as to why the medication is necessary for the patient. Additional patient requirements may include urine toxicology panels, urine pregnancy tests, or a consent form by which the patient pledges adherence to medication therapy and follow-up appointments. This added layer of approvals imposed by some third-party payers requires dedicated resources that may not be taken into account by many cost-effectiveness models [28]. Restrictions may also apply for severity of infection, which is commonly characterized by the METAVIR score that assesses liver necroinflammation and fibrosis. One study indicated that among the 42 states with known Medicaid restriction criteria for SOF, $74 \%$ limit treatment to patients with the highest METAVIR scores of F3 or F4 [29]. There is limited evidence to support some of the aforementioned requirements [1]. However, with more widespread use of DAA-containing regimens, there may be more evidence in the future to support or refute HCV "stewardship," such as rationing new agents for difficult patients and inexpensive agents for less complex patients. Notably, many of the logistical issues described above are unique to the US and may not be germane to other 
geographic locales, or in the future with movement towards universal healthcare coverage. As more $\mathrm{HCV}$-infected patients obtain access to treatment, infection rates by transmission may decline and subsequently decrease the overall societal and financial burden of HCV. An ideal regimen would be affordable and accessible for all patients seeking treatment.

\section{HIGH BARRIER TO RESISTANCE}

High efficacy demonstrated by several HCV regimens means treatment success for many patients. However, in the event of relapse or treatment failure, an ideal therapy would exhibit a high barrier to resistance with little potential for cross-resistance with other agents. Some regimens are affected by baseline NS5A mutations. Among GT1A-infected patients receiving 12 weeks of GZR/EBR, SVR was lower among patients with at least one baseline NS5A resistance-associated polymorphism at amino acid positions 28, 30, 31 or 93 [3, 9]. Thus, patients with GT1A infection initiating GZR/ EBR need to undergo NS5A testing. The presence of any of these four polymorphisms extends therapy from 12 to 16 weeks and requires the addition of ribavirin $[3,9]$. As the use of NS5A inhibitors becomes more ubiquitous and the issues of cross-resistance and persistence of NS5A and NS3 mutations are better understood, the impact of this test may become applicable to other treatment regimens. Findings may steer certain patient populations from using these therapies. Cross-resistance exists for some available agents including the protease inhibitors, SIM and paritaprevir $[7,11]$. SOF is advantageous in that it exhibits a high barrier to resistance and, when used in combination with other DAA agents, may still be used successfully to overcome the presence of baseline antiviral resistance-associated variants (RAV) $[9,16]$. LDV/SOF may offer a promising treatment option for patients who have failed a RBV-containing regimen or SIM/ SOF (though addition of RBV to LDV/SOF is recommended for the latter); however, data are limited [9]. PEG-IFN, though no longer a component of most preferred regimens, maintains activity in the setting of RAVs and therefore remains a viable adjunctive option for many treatment-experienced patients [1].

\section{FUTURE DIRECTIONS}

Several promising $\mathrm{HCV}$ treatment regimens with "ideal" traits lie just over the horizon. A novel NS5A inhibitor, velpatasvir (GS-5816), in combination with SOF for 12 weeks, has produced SVR12 rates $>90 \%$ in patients with GT1 through 6 in various stages of clinical study $[26,30]$. Cure was still achieved in most patients exhibiting baseline genetic viral variants for NS5A. While larger studies including more difficult-to-treat populations are needed, such as those with cirrhosis and history of treatment failure, preliminary results are encouraging [27]. A pangenotypic option, if possessing other traits of an ideal regimen, could increase accessibility of treatment for all HCV-infected patients, particularly those with currently limited treatment options based on specific genotypes. In addition to regimens offering broad-spectrum genotypic activity, shorter treatment durations are also being pursued. Several 4- and 6-week combination therapy regimens (2-3 agents) are being explored in phase II studies that will hopefully add additional highly efficacious, multigenotypic therapies to the growing HCV treatment armamentarium [30]. Though further beyond 
the horizon, emergence of generic treatment options will likely alter the treatment landscape once again. A series of questions will arise pertaining to cost-effectiveness of branded single-tablet regimens versus less expensive multiple-tablet regimens and the impact on regimen adherence and ultimate treatment success.

\section{CONCLUSIONS}

As the treatment landscape for chronic HCV infection continues to rapidly evolve, the characteristics associated with an ideal regimen remain constant. An ideal regimen is one that is efficacious in a variety of populations, convenient, safe, accessible/ affordable, and has a high barrier to resistance. Although significant progress has been made, no commercially available regimen fully achieves each of these desirable characteristics. It is imperative for continued research and development to achieve these goals to produce dramatic reductions in $\mathrm{HCV}$ infection burden globally.

\section{ACKNOWLEDGMENTS}

No funding or sponsorship was received for this study or publication of this article. This article has greatly benefited from the thoughtful content editing of John Caswell and Sarah Hoffman. All named authors meet the International Committee of Medical Journal Editors (ICMJE) criteria for authorship for this manuscript, take responsibility for the integrity of the work as a whole, and have given final approval for the version to be published.

Compliance with Ethics Guidelines. This article is based on previously conducted studies and does not involve any new studies of human or animal subjects performed by any of the authors.

Disclosures. N. Patel has received research grant support from Gilead Sciences and Merck. M. R. Bidell, M. McLaughlin, J. Faragon and C. Morse declare no pertinent conflicts of interest.

Open Access. This article is distributed under the terms of the Creative Commons Attribution-NonCommercial 4.0 International License (http://creativecommons.org/licenses/ by-nc/4.0/), which permits any noncommercial use, distribution, and reproduction in any medium, provided you give appropriate credit to the original author(s) and the source, provide a link to the Creative Commons license, and indicate if changes were made.

\section{REFERENCES}

1. Kohli A, Kapoor R, Sims Z, et al. Ledipasvir and sofosbuvir for hepatitis C genotype 4: a proof-of-concept, single-centre, open-label phase 2a cohort study. Lancet Infect Dis. 2015;15(9):1049-54.

2. Holmberg SD, Spradling PR, Moorman AC, Denniston MM. Hepatitis C in the United States. N Engl J Med. 2013;368(20):1859-61.

3. Lawitz E, Mangia A, Wyles D, et al. Sofosbuvir for previously untreated chronic hepatitis $\mathrm{C}$ infection. N Engl J Med. 2013;368(20):1878-87.

4. Martinot-Peignoux M, Stern C, Maylin S, et al. Twelve weeks posttreatment follow-up is as relevant as 24 weeks to determine the sustained virologic response in patients with hepatitis $C$ virus receiving pegylated interferon and ribavirin. Hepatology. 2010;51(4):1122-6.

5. Yoshida EM, Sulkowski MS, Gane EJ, et al. Concordance of sustained virological response 4 , 12, and 24 weeks post-treatment with sofosbuvir-containing regimens for hepatitis C virus. Hepatology. 2015;61(1):41-5.

6. Aghemo A, Rumi MG, Monico S, et al. The pattern of pegylated interferon-alpha2b and ribavirin 
treatment failure in cirrhotic patients depends on hepatitis $\mathrm{C}$ virus genotype. Antivir Ther. 2009;14(4):577-84.

7. Di L. The role of drug metabolizing enzymes in clearance. Expert Opin Drug Metab Toxicol. 2014;10(3):379-93.

8. Lawitz E, Sulkowski MS, Ghalib R, et al. Simeprevir plus sofosbuvir, with or without ribavirin, to treat chronic infection with hepatitis $C$ virus genotype 1 in non-responders to pegylated interferon and ribavirin and treatment-naive patients: the COSMOS randomised study. Lancet. 2014;384(9956):1756-65.

9. Zahnd C, Salazar-Vizcaya LP, Dufour JF, Swiss HIV and Hepatitis C Cohort Studies Team, et al. Impact of deferring $\mathrm{HCV}$ treatment on liver-related events in HIV+ patients. Abstract 150. Conference on retroviruses and opportunistic infections. Seattle, February 23-24, 2015.

10. Gritsenko D, Hughes G. Ledipasvir/sofosbuvir (harvoni): improving options for hepatitis $\mathrm{C}$ virus infection. P T. 2015;40(4):256-76.

11. Beasley D, Humer C. Payers see price leverage with entry of Merck hepatitis C drug. Reuters US January 29, 2016.

12. Gill K, Ghazinian H, Manch R, Gish R. Hepatitis C virus as a systemic disease: reaching beyond the liver. Hepatol Int. 2015;10(3):415-23.

13. Stepanova $M$, Younossi ZM. Interferon-free regimens for chronic hepatitis $\mathrm{C}$ : barriers due to treatment candidacy and insurance coverage. Dig Dis Sci. 2015;60(11):3248-51.

14. Fontaine $\mathrm{H}$, Lazarus A, Pol $\mathrm{S}$, et al. Bradyarrhythmias associated with sofosbuvir treatment. N Engl J Med. 2015;373(19):1886-8.

15. Brainard DM, McHutchison JG. Bradyarrhythmias associated with sofosbuvir treatment. N Engl J Med. 2015;373(19):1888.

16. Feld JJ, Jacobson IM, Hezode C, et al. Sofosbuvir and velpatasvir for HCV genotype 1, 2, 4, 5, and 6 infection. N Engl J Med. 2015;373(27):2599-607.

17. Fissell RB, Karaboyas A, Bieber BA, et al. Phosphate binder pill burden, patient-reported non-adherence, and mineral bone disorder markers: findings from the DOPPS. Hemodial Int. 2016;20(1):38-49.

18. Hanna DB, Hessol NA, Golub ET, et al. Increase in single-tablet regimen use and associated improvements in adherence-related outcomes in
HIV-infected women. J Acquir Immune Defic Syndr. 2014;65(5):587-96.

19. Libby AM, Fish DN, Hosokawa PW, et al. Patient-level medication regimen complexity across populations with chronic disease. Clin Ther. 2013;35(4):385-98 (e381).

20. Cohen CJ, Meyers JL, Davis KL. Association between daily antiretroviral pill burden and treatment adherence, hospitalisation risk, and other healthcare utilisation and costs in a US medicaid population with HIV. BMJ Open. 2013;3(8):1-12.

21. Scott Sutton S, Magagnoli J, Hardin JW. Impact of pill burden on adherence, risk of hospitalization, and viral suppression in patients with HIV infection and AIDS receiving antiretroviral therapy. Pharmacotherapy. 2016;36(4):385-401.

22. Sutton SS, Hardin JW, Bramley TJ, D'Souza AO, Bennett CL. Single- versus multiple-tablet HIV regimens: adherence and hospitalization risks. Am J Manag Care. 2016;22(4):242-8.

23. Kowdley KV, Gordon SC, Reddy KR, et al. Ledipasvir and sofosbuvir for 8 or 12 weeks for chronic $\mathrm{HCV}$ without cirrhosis. N Engl J Med. 2014;370(20):1879-88.

24. Kwo P, Gitlin N, Nahass R, et al. Simeprevir plus sofosbuvir (12 and 8 weeks) in HCV genotype 1-infected patients without cirrhosis: OPTIMIST-1, a phase 3, randomized study. Hepatology. 2016. doi:10.1002/hep.28467.

25. Sulkowski M, Hezode C, Gerstoft J, et al. Efficacy and safety of 8 weeks versus 12 weeks of treatment with grazoprevir (MK-5172) and elbasvir (MK-8742) with or without ribavirin in patients with hepatitis $\mathrm{C}$ virus genotype 1 mono-infection and HIV/hepatitis C virus co-infection (C-WORTHY): a randomised, open-label phase 2 trial. Lancet. 2015;385(9973):1087-97.

26. Everson GT, Towner WJ, Davis MN, et al. Sofosbuvir with velpatasvir in treatment-naive noncirrhotic patients with genotype 1 to 6 hepatitis $C$ virus infection: a randomized trial. Ann Intern Med. $2015 ; 163(11): 818-26$.

27. Curry MP, O'Leary JG, Bzowej N, et al. Sofosbuvir and velpatasvir for $\mathrm{HCV}$ in patients with decompensated cirrhosis. $\mathrm{N}$ Engl J Med. 2015;373(27):2618-28.

28. Chhatwal J, Kanwal F, Roberts MS, Dunn MA. Cost-effectiveness and budget impact of hepatitis $\mathrm{C}$ virus treatment with sofosbuvir and ledipasvir in the United States. Ann Intern Med. 2015;162(6):397-406. 
29. Barua S, Greenwald R, Grebely J, Dore GJ, Swan T, Taylor LE. Restrictions for medicaid reimbursement of sofosbuvir for the treatment of hepatitis $C$ virus infection in the United States. Ann Intern Med. 2015;163(3):215-23.
30. Dore GJ, Feld JJ. Hepatitis C virus therapeutic development: in pursuit of "perfectovir". Clin Infect Dis. 2015;60(12):1829-36. 